
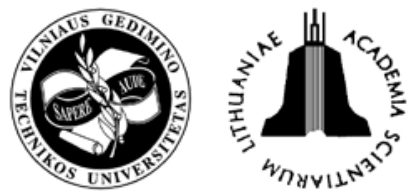

TRANSPORT

2009

24(1): $21-25$

\title{
DEVELOPING CO-OPERATIVE TRANSPORT SYSTEM AND ROUTE PLANNING
}

\author{
Gábor Szücs \\ Dept of Telecommunications and Media Informatics, Budapest University of Technology and Economics, \\ 2nd Magyar Tudósok Krt., H-1117, Bld. I. B. Room 222, Budapest, Hungary \\ E-mail:szucs@tmit.bme.hu
}

Received 15 September 2008; accepted 12 January 2009

\begin{abstract}
This paper consists of two parts: planning Co-operative Transport Systems (CTS) with general rules (communication is a new idea in this system) and an investigation into route planning as a part of services provided in CTS. Part one reveals developing new systems for cooperative sensing and predicting flow, infrastructure and environmental conditions surrounding traffic, with a view to improve the safety and efficiency of road transport operations. Part two introduces the enhancement of the performance of the route planning algorithms. The key issues of these parts are communication and co-operation between the elements of the whole Co-operative Transport System.
\end{abstract}

Keywords: eSafety, intelligent transport system, communication, co-operation, Dijkstra algorithm, speed-up techniques.

\section{Introduction}

A high number of people are annually killed, injured or become disabled in road traffic crashes. The causes of the accidents may vary but can be classified considering different aspects because road infrastructure, a type of the vehicle, the driver and their interactions are covering the major groups of these causes. An important task is to find bottlenecks where the potential of danger is high and where a system rather than the already accepted traditional signals should give the driver a direct notice of such risk.

\section{Transportation Systems}

\section{1. eSafety}

The objective of the eSafety initiative is to accelerate the development, deployment and use of new technologies for increasing road safety. It was launched at the eSafety high-level meeting and is a joint industry-public sector initiative. The report was made of 28 recommendations one of which was standardization adopted by the Commission Communication 'ICT for safe and intelligent vehicles'. The document discloses that one 'cannot rely on voluntary actions' as it is necessary to promote intelligent vehicle communication. The eSafety forum continues and has some industry-led working groups:

- in-vehicle emergency call (eCall) - an integrated strategy for a pan-European emergency service,
- human-machine interaction - in-vehicle eSafety systems,

- research and development - focus on further research,

- real time traffic and travel information.

Upon the completion of the research block of the proposal, recommendations will be formulated for a further development of communication devices to enhance safety following the targets of the eSafety forum. New technology requirements will be defined, e.g. how to build and implement self-configuring communication networks including their reaction to an automatically created deviation plan for an obstacle arising from an accident.

The provision of information to influence traffic flow management in case of accident can be used for simpler tasks. The access management based on this information may be used for optimizing the long route planning - communication tools, ways and knowledge will be in part already available. eSafety (with a new service: eCall) may give new impetus for safe lives in the roads. eCall (communication possibility in the vehicle) may solve a series of communication problems actually not to be seen because of the non existence of co-operation between the infrastructure and the vehicle (for example, see researches by Jarašūnienè and Jakubauskas 2007; Batarlienè and Baublys 2007; Yousefi and Fathy 2008). 


\subsection{Intelligent Transport System Architectures}

Intelligent Transport Systems (ITS) frequently span several transport means (e.g. private and public transport) and are provided in many locations (on board vehicles, at the roadside, at home, at the office or through mobile devices). The use of ITS architecture also makes possible to highlight any problems that arise from the refinement and modification of the services being provided. The architecture can become a 'tool' that enables traffic and transport problems to be addressed and resolved.

The first version of the European ITS Framework Architecture was published in 2000. The content was developed by means of constant consultation on intermediate results with European Stakeholders and a panel of Wise-men. The final outcome was agreed and endorsed by the major European actors as the Framework Architecture to be used as the reference providing:

- the ability of travelers and freight operators to plan and implement journeys using the most efficient, convenient and cost-effective combinations of transport modes;

- compatibility and consistency of information delivered to end-users through different media (any end-user should be able to receive the same information through different media such as GSM, etc.);

- compatibility of equipment with different infrastructures, thus enabling 'seamless' travel across Europe (e.g. a driver should be able to use the in-vehicle system for electronic payment both at home and abroad);

- a basis for regional, national or European authorities to produce master plans and recommendations to facilitate ITS deployment allowing, for instance, authorities to co-operate on traffic management across borders and boundaries;

- an open market for services and equipment where compatible sub-systems are offered (no more adhoc solutions);

- economies of scale in equipment manufacture permitting competitive prices and cheaper investments with compatibility guaranteed;

- a known market place into which producers can supply products with reduced financial risk.

Within Europe, the new applications of ITS are being continually developed. It is crucial to ensure that their deployment is coordinated in a way that ensures the overall and long term improvement of transport efficiency. Without a common European ITS Framework Architecture, there is a risk that products providing ITS services will be developed in isolation, and therefore will not be able to easily interact with each other. It may also become impossible for a consistent level of service to be uniformly provided to citizens throughout Europe. It is therefore essential for all parties involved in the implementation of ITS across Europe to adhere to an agreed common Framework Architecture that must accommodate national plans and support efforts in research, standardization, deployment and investment. It must also ensure that new systems are not only compatible but also include 'migration' plans for the incorporation and adaptation of existing or 'legacy' installations.

\subsection{Co-operative Transport Systems}

The problem of safety in transport is especially acute in the case of road networks. On the other hand, there are many safety standards in Europe and all over the world. Existing infrastructure will need to be operated up to (and sometimes beyond) its design margins in order to cope with the additional capacity. However, in this case, safety standards must not only be maintained but also increased at current levels. The safety is based on autonomous or stand-alone systems, and there is no possibility of reaching a high level safety without cooperation among the stand-alone systems, so the improvement is limited. In order to overcome the visible limitations of the previously discussed systems, there is a need for another type of solution: Co-operative Transport Systems (CTS) the intelligence of which is distributed/shared between road infrastructure and vehicles are compromising innovative approaches on transport safety and efficiency. Undoubtedly, the currently available (or under development) technologies enable improving not only sensing systems but also the way these systems share information. Smarter systems demand smarter sensors and cooperative systems require cooperative sensors. The above mentioned idea can be improved and developed by a number of breakthrough sensing technologies and a new model for knowledge sharing between sensors.

In this context, stand-alone Intelligent Transport Systems (ITS) are not the solution any longer. Instead, Co-operative Transport Systems (CTS) are the most promising option. In this paradigm, analogous to that of the Ambient Intelligence, different actors involved in ITS - humans and machines - will interact among each other in order to meet their individual goals while at the same time maximizing the safety and efficiency of the overall system. The systems which can deliver smarter functionalities are needed.

When summarizing the above mentioned aims, the objectives are as follows: to contribute towards improving road safety, optimize the efficiency of the road network, improve the road transport environment, establish new advanced sensing systems and integrate all of them into CTS. It is also increasingly important that they should be fully compatible not only within a single country but also at the international level.

\subsection{Planning and Impact of CTS}

The goal can be achieved through several work-phases the first of which is innovation in sensing technologies. The number of sensing technologies can be divided into two parts: on-board vehicle sensors and external sensing technologies.

The next stage is embedding sensing technologies developed into the CTS environment. It is important to enable the modular integration of the sensors into the CTS architecture to be developed and adopted within eSafety Programme. 
An optional possibility of making the system more complex is creating a knowledge based Decision Support System to assess and predict the ambient conditions affecting the safety and efficiency of transport.

The system with innovative sensing technologies will improve road safety and efficiency in several main aspects:

- will help with raising the in-vehicle awareness of external conditions (other vehicles, environment, infrastructure etc.);

- will assist in assessing general traffic conditions even in complex situations where many different actors - humans and machines - are interacting;

- will enable to take more complex decisions in a distributed manner by using better and more comprehensive information and knowledge.

The communication mechanisms will be intended to be compatible with the other protocols and architectures in the eSafety Programme.

\section{TRACKSS Project Dealing with Cooperation}

TRACKSS (Technologies for Road Advanced Cooperative Knowledge Sharing Sensors) is the EU project that was launched in 2006 and will be completed in 2009 . (TRACKSS project documents 2006). The aim of the project is the support of network planning, road traffic modelling, management, monitoring and control. Synchronised with the eSafety programme, the project will have essential effects on more factors of safety and hopefully on statistics on fatalities accidents.

These were the global aims, and in order to reach this purpose, the task is working towards an effective solution which is Co-operative Transport Systems (CTS) the intelligence of which is distributed among the vehicles and the road infrastructure (Szücs 2007).

The TRACKSS will contribute to and seek compatibility with pan-European e-Safety architecture. The consortium developing this ambitious project counts up 15 partners including global industrial players, innovative SME's and world-class research institutions from the UK, Spain, France, Germany, Italy, Hungary and the Czech Republic. The objective of the CTS can be seen in the Figure 1 where the situation before and after the project is shown. The knowledge sharing mechanism among different CTS elements can be seen in the Figure 2. The new idea
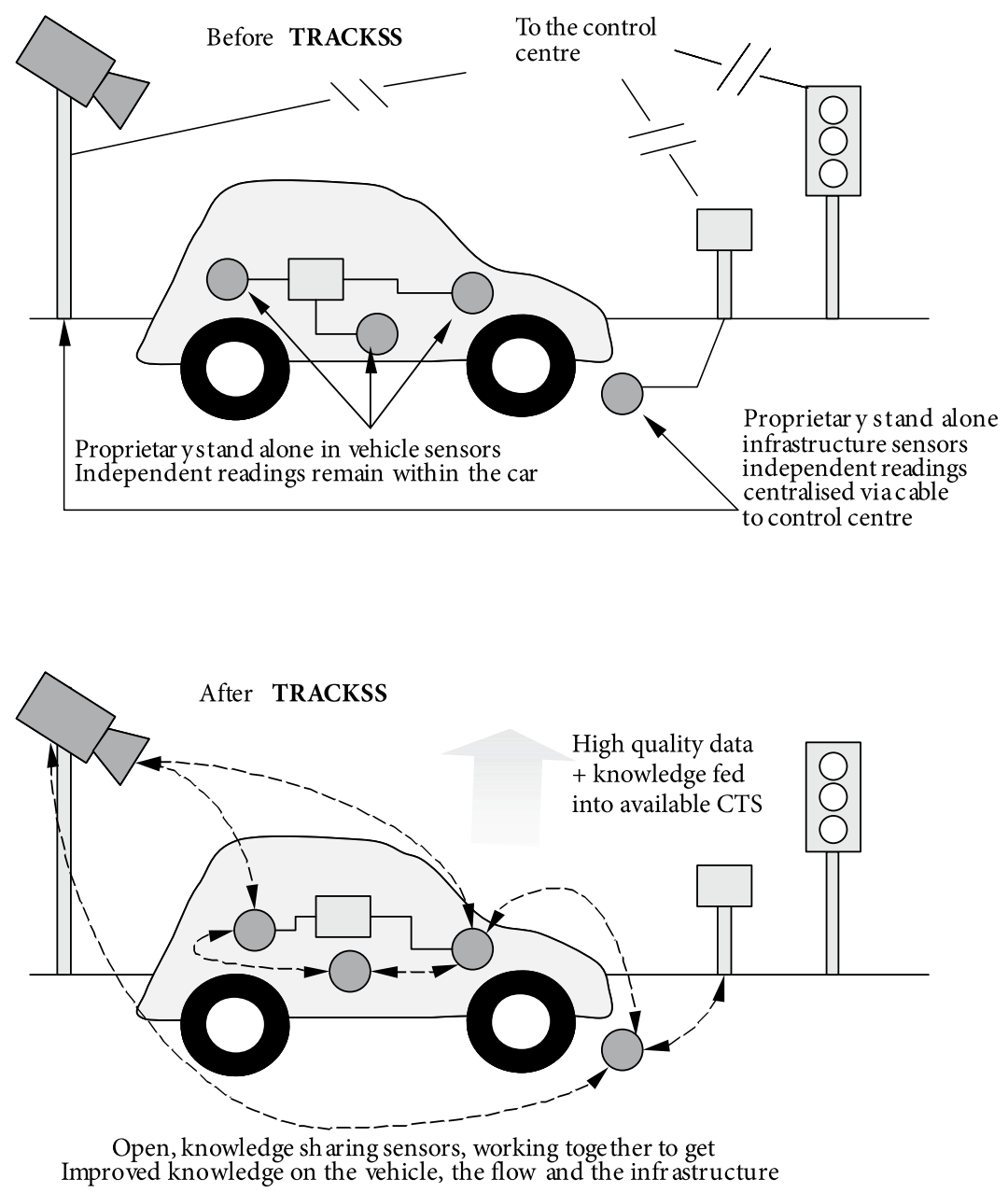

Fig. 1. The solutions to transport systems before and after the TRACKSS 


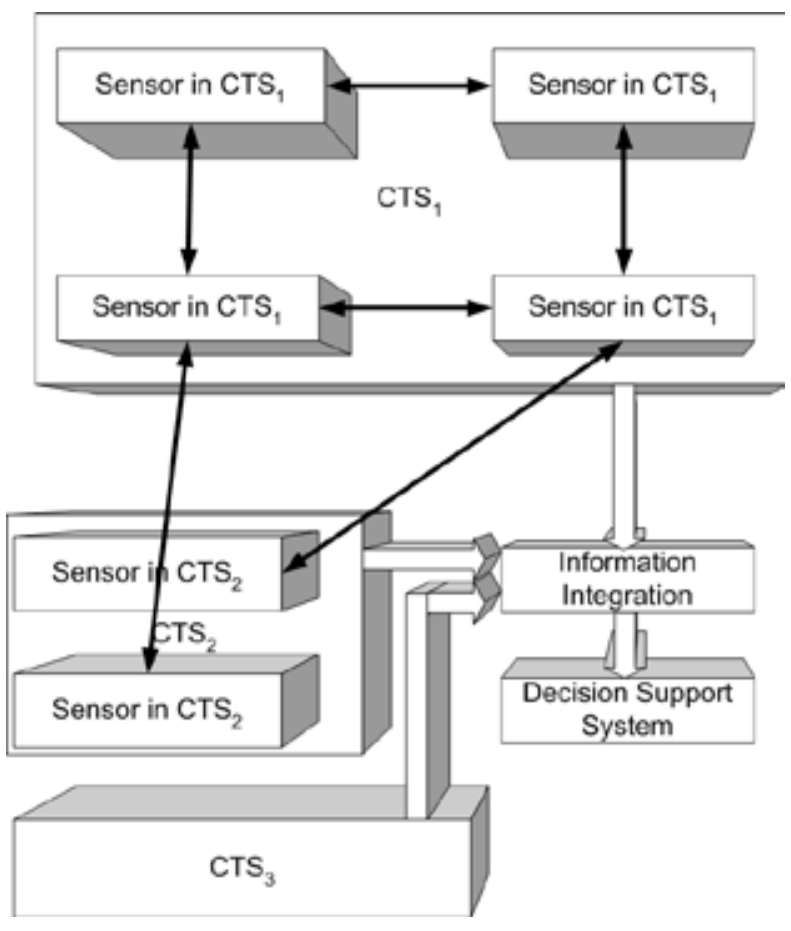

Fig. 2. Knowledge Sharing among different CTS elements

is knowledge sharing (Marqués 2007) not only among the components of a CTS but also among all elements in different CTS systems (like in the Figure 2).

\section{Route Planning}

Co-operative Transport Systems (CTS) are useful not only for traffic safety but also for traffic services like route planning. In a CTS, the road network (as a part of the infrastructure) can be represented a graph. There are some shortest path algorithms that allow fast point-to-point queries in graphs using preprocessed data. A standard algorithm for this problem is the one developed by $\mathrm{Di}$ jkstra algorithm (Dijkstra 1959). Although this standard algorithm is fast in the theory, the corresponding algorithms are often not fast enough for applications in the large networks that require a huge number of the shortest path computations. A speed-up technique is needed, and therefore some algorithms (Goldberg and Werneck 2005; Delling and Wagner 2007) like bidirectional Dijkstra, $\mathrm{A}^{\star}$ algorithm, Arc-Flags, Highway Hierarchies have been developed to solve this problem.

The most known speed-up technique of the search is the bidirectional Dijkstra algorithm. In this procedure, an additional search is started from the target node and the query stops as soon as both searches meet. Applying this approach, the paths of forward and backward search are alternated. The reason for this approach is that two searches has lower complexity: $\mathrm{O}(\mathrm{bd} / 2+\mathrm{bd} / 2$ ) is much less than the running time of one search from start to finish which would be $\mathrm{O}(\mathrm{bd})$.

The $A^{*}$ algorithm (Goldberg and Harrelson 2005; Hart et al. 1968) (as a goal directed search) pushes the search towards a target by adding a potential to the priority of each node. Given a 2-dimensional layout, the usage of Euclidean potentials requires no preprocessing. The algorithm uses the distance-plus-cost heuristic function to determine the order in which the search visits nodes in the tree. The distance-plus-cost heuristic is a sum of two functions: the path-cost function (which may or may not be a heuristic) and an admissible 'heuristic estimate' of the distance to the goal. The path-cost function is the cost from the starting node to the current node.

The Arc-Flags (Möhring et al. 2005; Köhler et al. 2006) method is a modified Dijkstra algorithm in order to avoid exploring unnecessary paths. This means the procedure checks the flag entry of the corresponding target region (the region where the target node $t$ belongs to) every time before the Dijkstra algorithm wants to traverse an arc. This is the only modification to the standard Dijsktra algorithm. More precisely, the Arc-Flag approach partitions the graph into cells and attaches a label to each edge. A label contains a flag for each cell indicating whether the shortest path to the corresponding cell exists that starts with this edge. As a result, Arc-Flag Dijkstra often only visits those edges which lie on the shortest path of a long-range query. Thus, implementing the arc-flags is one of the easiest acceleration modifications of the standard Dijkstra algorithm known.

Highway Hierarchies (Sanders and Schultes 2006) uses the concept of local search. This approach is a purely hierarchical method, i.e. an approach trying to exploit the hierarchy of a graph. Therefore, the network is contracted and then 'important' edges (the so called highway edges) are identified. By rerunning those two steps, a natural hierarchy of the network is obtained. The contraction phase builds the core of a level and adds shortcuts to the graph. The identification of highway edges is done by local Dijkstra executions.

These speed-up techniques can be used in a large road network. It is an important issue in CTS systems, because Co-operative Transport Systems can be implemented from several Intelligent Transport Systems and the infrastructure is the union set of these standalone systems, so the network can be very large

\section{Conclusions}

The elements of the co-operation among operators, infrastructure, vehicles, their drivers and other road users will be established and supported with the aim to deliver a more efficient, safe, secure and comfortable journey as summarized by the goals of the CTS. The essence of the co-operative systems is that they involve two-way communications with real-time interactions. The vehicle may communicate directly with one another, so that a driver knows, for example, if another vehicle is on a conflict course. Vehicles may also provide data directly to traffic management and receive individual guidance and in support return. Essentially, these systems can help in route planning and greatly extend the driver's range of perception providing relevant information on the behaviour of traffic in general and neighbouring vehicles in particular beyond the range of vision. 
After the development of the whole CTS system, a thorough analysis should be executed. The aim of analysis based on real data is the diminution of the number and consequences of accidents. The platform for data exchange and investigation processes should be established in regional or national frame containing information on traffic management and national and local road network including quality and regulation.

This paper has shown new cooperative possibilities that will provide a new approach for transport systems making them cooperative by developing a brand new set of safety functions and new devices. Using cooperative sensors, we will get more detailed information about circumstances that can have large benefit to critical situations and route planning.

\section{References}

Batarlienė, N.; Baublys, A. 2007. Mobile solutions in road transport, Transport 22(1): 55-60.

Delling, D.; Wagner, D. 2007. Landmark-based routing in dynamic graphs, Lecture Notes in Computer Science (Experimental Algorithms 6th International Workshop, WEA 2007) 4525: 52-65.

Dijkstra, E. W. 1959. A note on two problems in connexion with graphs, Numerische Mathematik 1: 269-271.

Goldberg, A. V.; Harrelson, C. 2005. Computing the shortest path: $\mathrm{A}^{*}$ meets graph theory, in 16th Annual ACM-SIAM Symposium on Discrete Algorithms (SODA '05), 156-165.

Goldberg, A. V.; Werneck, R. F. 2005. An efficient external memory shortest path algorithm, in 7th Workshop on Algorithm Engineering and Experiments (ALENEX'05), 26-40.

Hart, P. E.; Nilsson, N. J.; Raphael, B. 1968. A formal basis for the heuristic determination of minimum cost paths, IEEE Transactions on Systems Science and Cybernetics 4(2): 100-107.

Jarašūnienè, A.; Jakubauskas, G. 2007. Improvement of road safety using passive and active intelligent vehicle safety systems, Transport 22(4): 284-289.

Köhler, E.; Möhring, R. H.; Schilling, H. 2006. Fast point-to-point shortest path computation with arc-flags, in 9th DIMACS Challenge on Shortest Paths. 27 p. Available from Internet: <http:// www.dis.uniroma1.it/ challenge9/papers/kohler.pdf $>$.

Marqués, A. 2007. Cooperative sensors making use of a common knowledge sharing model, in Proceedings of ITS Word Congress, Beijing, China, October.

Möhring, R. H.; Schilling, H.; Schütz, B.; Wagner, D.; Willhalm, T. 2005. Partitioning graphs to speed up Dijkstra's algorithm, Lecture Notes in Computer Science (Experimental and Efficient Algorithms, 4th International Workshop, WEA 2005) 3503: 189-202.

Sanders, P.; Schultes, D. 2006. Engineering highway hierarchies, in Lecture Notes in Computer Science (Algorithms - ESA 2006, 14th Annual European Symposium) 4168: 804-816.

Szücs, G. 2007. Kooperatív közlekedési rendszereknemzetközi megoldásai TRACKSS projekt kapcsán [International Solutions of Co-operative Transport Systems Apropos of TRACKSS Project], Közlekedéstudományi Szemle LVII: 466-470.

TRACKSS project documents. 2006. Website of consortium 'Technologies for road advanced cooperative knowledge sharing sensors. Available from Internet: <www.trackss.net>.

Yousefi, S.; Fathy, M. 2008. Metrics for performance evaluation of safety applications in vehicular ad hoc networks, Transport 23(4): 291-298. 